\title{
Research on Online Assessment of Switch Resistance Based on Real-time Current Measurement
}

\author{
Jiankun Liu \\ Jiangsu Electric Power Company Research Institute \\ Nanjing,China
}

Jing $\mathrm{Bu}$ (corresponding author)

Nanjing University of Science \& Technology

Nanjing,China

bujing30@foxmail.com

Qian Zhou

Jiangsu Electric Power Company Research Institute Nanjing,China

\author{
Chenggen Wang \\ Jiangsu Electric Power Company Research Institute \\ Nanjing,China \\ Yang Hou \\ Nanjing University of Science \& Technology \\ Nanjing,China \\ Minghui Yin \\ Nanjing University of Science \& Technology \\ Nanjing,China
}

\begin{abstract}
The evaluation of switch resistance has aroused widespread concern.To assess the resistance of switchgear in switching substations which use $3 / 2$ wiring, online assessment method based on real-time current measurements is proposed in this paper. The method use Kirchhoff's law to establish the mathematical model of switching substations which use $3 / 2$ wiring. It calculates the resistance of switchgear as finding solutions of non-homogeneous linear equations. The minimum number of current to be measured is determined by these equations. We can get a lot of current conditions in real-time from the current transformer.We can derive the required current conditions to establishe the model so we can get the answer. Finally, this article uses operating data of a $500 \mathrm{kV}$ substation to calculate the resistance of switchgear. The simulation results reflect the effectiveness of the algorithm Comparing with the traditional method, this method is easier and more accurate.SO an effective way is provided To assess the switch resistance
\end{abstract}

Keywords - online assessment; Real-time Current ; switch resistance; power system; 3/2 wiring

\section{INTRODUCTION}

Currently,500kV substations bear the assignment including collecting energy, redistributing load and transporting power. As the high operational reliability, $3 / 2$ wiring has been the mainstream wiring of $500 \mathrm{kV}$ substations. The condition monitoring of switching equipment is a prerequisite to achieve predictable maintenance and a key to ensure electrical equipment's safety. Also, it's a major complement and a new development to traditional offline preventive tests. So, it's important to assess switch online for detecting faults, hazards and maintaining the normal operation of the transformer substation.

Off-line and on-line are the main methods to measure the switch resistance. There are a lot of methods to measure the switch resistance off-line. Isao Minowa who comes from Japan uses superconducting quantum device to measure the switch resistance[1]. H.Aiclli measures the switch by cell method[2], while Polish scholar JerzvKaczmarek measures the switch by the third harmonic[3]. These methods are based on the theoretical study of electrical contacts in the laboratory. M.landry proposes a method to measure the dynamic contact resistance[4-8] and Li Kui measures the switch resistance by pulse current $[9-10]$. This method can only measure the purely resistive element, although it improves the accuracy effectively. The traditional method is using DC double bridge in engineering application and the current is small in measurement loop[11]. It's slow and inaccurate to use this method as it needs to adjust the bridge manually. Now the switch resistance is usually measured by microhm instrument[12]. But this method lower the measurement's accuracy and it's inconvenient in field test for the big volume and the high weight. For requirements of online measurement, article [13] proposed a method to calculate the steady-state temperature rise by measuring the circuit breaker housing temperature and the ambient air temperature. Then, the main contact loop resistance can be calculated indirectly according to the steady-state temperature. This method must be used in steady state and the measurement error is large. Article [14] evaluates the status of DC circuit breaker by analyzing the current waveform of oscillation circuit in HVDC circuit breaker with genetic algorithms. It's accurate and reliable.

To solve the problems above, this paper proposes a method which based on real-time current measurement to calculate the switch resistance in $3 / 2$ wiring switching substation. It assesses the switch status using 
corresponding circuit theory and algorithms by measuring the real-time current in the substation.

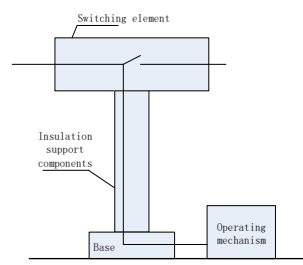

(a)Shell charged

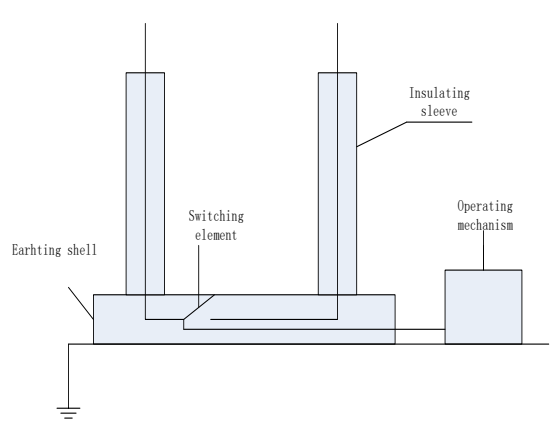

(b)enclosure grounding

Figure 1.Typical structure of high voltage circuit breaker

\section{THE STRUCTURE OF SWITCHGEAR}

Switchgear in the switching station includes circuit breaker and isolating switch. The basic structure of circuit breaker and isolating switch is the same. And it is composed of operating mechanism, transmission mechanism, circuit breaking device, insulation support components and base. High voltage circuit breaker mainly divides into the shell charged type and enclosure grounding type. The typical structure is shown in Fig .1[15].

The movements of switchgear are controlled by the operating system. The operator sends instruction to the operating mechanism. Then Operating Mechanism controls Transmission Mechanism to make the switch on and off. The operating system structure of switchgear is shown in Fig .2. The Lift Mechanism and the Drive Transmission mechanism is the transmission part between moving contact and operating mechanism. Transmission mechanism is needed to connect lifting mechanism and operating mechanism. Not only because of the interval between them, but also the different moving direction. Transmission mechanism controls the moving contact. Lift Mechanism can make switch contact move in a straight line or approximate straight-line[16].

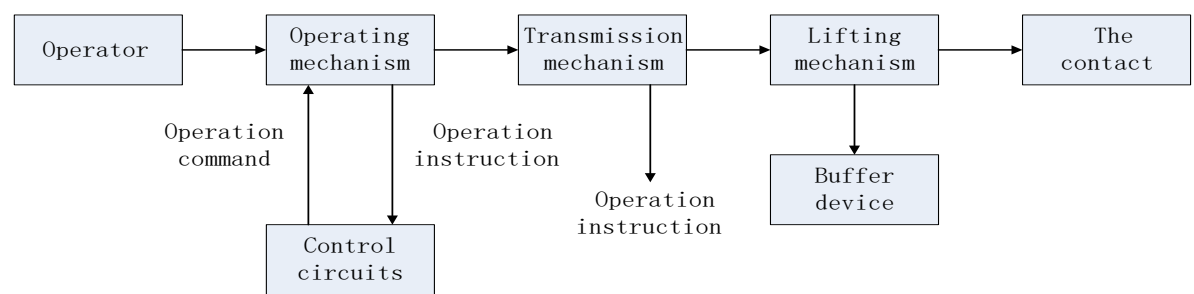

Figure 2. The operating system structure of switchgear

\section{ASSESSMENT METHOD OF SWITCH RESISTANCE}

\section{A. Loop resistance}

Loop resistance of switchgear is mainly contact resistance between static and dynamic contact. The contact resistance is mainly determined by conductivity of the material and geometry of the switch. The loop resistance will increase obviously after long time use of switchgear. One reason is the mechanical cause. Frequent switching action makes the mechanical wear or mechanical failure of contacts. The contact area of contacts smaller, the contact resistance will become larger. Another reason is electrochemical factor. Large current for a long time and interrupters operation will cause the oxidation reaction of the contact. Oxide film covered contact surface will increase contact resistance.

The increased loop resistance will make the switch temperature rise sharply in a large current. It will shorten the service life of switchgear and threat the stability of power system. So the resistance of switchgear must be tested by manufacturers. Contact resistance of switchgear can be estimated by the following formula[17]:

$$
R_{h}=R_{d}+R_{j}
$$

where:

$$
\begin{aligned}
& R_{d}=\rho \frac{L}{S} \\
& R_{j}=K_{c} / F^{m}
\end{aligned}
$$

$\mathrm{Rh}$ is loop resistance of switchgear. $\mathrm{Rd}$ is resistance of the conductive material. $\mathrm{Rj}$ is contact resistances of switch. $\rho$ is the conductivity of conductive materials. L is the equivalent length of switch and $\mathrm{S}$ is the equivalent sectional area of switch. $\mathrm{Kc}$ is the coefficient related to the material surface and the contact way. $\mathrm{F}$ is the contact pressure. $\mathrm{m}$ is the coefficient related to contact way, it can be taken as 0.5 for point contact, 0.7 for line contact and 1 for surface contact.

The above formula can only make a rough estimate of switchgear resistance. Other methods must be used if precise calculation is needed. 


\section{B. Mathematical model}

Assuming the transformer substation has $\mathrm{m}$ branches of three-switches and $\mathrm{n}$ branches of two-switches. The diagram of $3 / 2$ wiring transformer substation is showed in Fig .3. The measured current of each switch in a moment is: $i_{1} i_{2} i_{3} \mathrm{~L} i_{3 m+2 n-1} i_{3 m+2 n}$, the resistance of each switch is: $R_{1} R_{2} R_{3} \mathrm{~L} R_{3 m+2 n-1} R_{3 m+2 n}$.

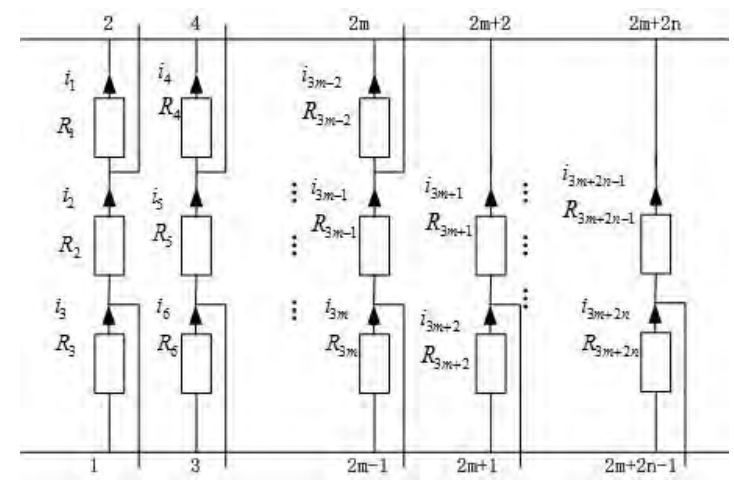

Figure 3. Diagram of 3/2 wiring transformer substation

It can be obtained from circuit theory:

Terminal voltage of the first branch:

$$
U_{12}=i_{1} \cdot R_{1}+i_{2} \cdot R_{2}+i_{3} \cdot R_{3}
$$

Terminal voltage of the second branch:

$$
U_{34}=i_{4} \cdot R_{4}+i_{5} \cdot R_{5}+i_{6} \cdot R_{6}
$$

Terminal voltage of the $m_{\mathrm{th}}$ branch:

$$
U_{2 m-1,2 m}=i_{3 m-2} \cdot R_{3 m-2}+i_{3 m-1} \cdot R_{3 m-1}+i_{3 m} \cdot R_{3 m}
$$

Terminal voltage of the $(m+n)_{\mathrm{th}}$ branch:

$$
U_{2 m+2 n-1,2 m+2 n}=i_{3 m+2 n-1} \cdot R_{3 m+2 n-1}+i_{3 m+2 n} \cdot R_{3 m+2 n}
$$

Ignoring the pressure drop on the bus:

$$
U_{12}=U_{34}=\mathrm{L}=U_{2 m-1,2 m}=U_{2 m+1,2 m+2}=\mathrm{L}=U_{2 m+2 n-1,2 m+2 n}
$$

Assuming $R_{1}$ is reference resistance, i.e., $a=1$, we can get:

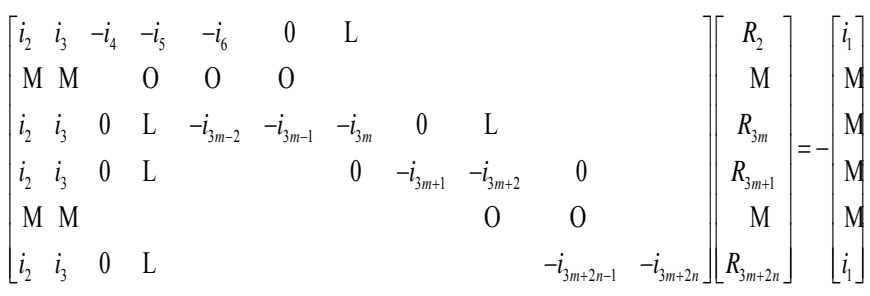

From above steps, we can solve linear equations (9) instead of calculating the switch resistance. The number of equations (9) is less than the number of unknowns.
Equations (9) can't be solved by a set of current conditions unless measuring different sets of current conditions. When the number of equations is more than the number of unknowns, we need to select some equations from all.

\section{Conditions Selection}

First of all, considering the least number of current conditions we need to measure to solve equations (9). $\mathrm{m}+\mathrm{n}-1$ equations can be established from a set of current conditions, while the number of unknowns is $3 m+2 n-1$. So, the least number of current conditions:

$$
N=\frac{3 m+2 n-1}{m+n-1}=2+\frac{m+1}{m+n-1}
$$

Usually, the number of three-switch branch is more than two: $m \geq 2$, the number of two-switch branch is more than zero: $n \geq 0$. So:

$$
N= \begin{cases}3 & n \geq 2 \\ 4 & 0 \leq n<2\end{cases}
$$

The least number of current conditions depends on the number of two-switch branch n. If $n \geq 2$, we only need to measure three sets of current conditions at least: $i_{1}^{(1)} \mathrm{L} i_{3 m+2 n}^{(1)}, i_{1}^{(2)} \mathrm{L} i_{3 m+2 n}^{(2)}, i_{1}^{(3)} \mathrm{L} i_{3 m+2 n}^{(3)}$. If $n<2$, we need to measure four sets of current conditions at least:

$$
i_{1}^{(1)} \mathrm{L} i_{3 m+2 n}^{(1)}, i_{1}^{(2)} \mathrm{L} i_{3 m+2 n}^{(2)}, i_{1}^{(3)} \mathrm{L} i_{3 m+2 n}^{(3)}, i_{1}^{(4)} \mathrm{L} i_{3 m+2 n}^{(4)}
$$

Then, equations (9) can be changed to the following equations:

$$
\left[\begin{array}{ccccccc}
i_{2}^{(1)} & i_{3}^{(1)} & -i_{4}^{(1)} & -i_{5}^{(1)} & -i_{6}^{(1)} & \mathrm{L} & 0 \\
\mathrm{M} & \mathrm{M} & & \mathrm{O} & \mathrm{O} & \mathrm{O} & \\
i_{2}^{(1)} & i_{3}^{(1)} & 0 & \mathrm{~L} & 0 & -i_{3 m+2 n-1}^{(1)} & -i_{3 m+2 n}^{(1)} \\
\mathrm{M} & & & & & & \\
i_{2}^{(N)} & i_{3}^{(N)} & -i_{4}^{(N)} & -i_{5}^{(N)} & -i_{6}^{(N)} & \mathrm{L} & 0 \\
\mathrm{M} & \mathrm{M} & & \mathrm{O} & \mathrm{O} & \mathrm{O} & \\
i_{2}^{(N)} & i_{3}^{(N)} & 0 & \mathrm{~L} & 0 & -i_{3 m+2 n-1}^{(N)} & -i_{3 m+2 n}^{(N)}
\end{array}\right]\left[\begin{array}{c}
R_{2} \\
\mathrm{M} \\
R_{3 m+2 n}
\end{array}\right]=-\left[\begin{array}{c}
i_{1}^{(1)} \\
\mathrm{M} \\
i_{1}^{(1)} \\
\mathrm{M} \\
i_{1}^{(N)} \\
\\
i_{1}^{(N)}
\end{array}\right](12)
$$

In general, the value of the current can be obtained from the current transformer installed in the switch station. The number of the condition can be measured is far more than four groups. Pick out the same number of the equations with the unknowns from these equations and simultaneous equations. Then solve them to get the ratio of each switch in the switching station.

\section{THE SIMULATION ANALYSIS}

To verify the effectiveness of the method, we can do a simulation by using the current data from a $500 \mathrm{kV}$ switching station of $3 / 2$ connection mode. Fig .4 is the wiring diagram of the switching station. Table 1 is the selected current data according to the operation of a quarter of the switching station. 
TABLE I. THE CURRENT DATA FLOWING THROUGH THE SWITCH AT DIFFERENT TIMES

\begin{tabular}{cccccccccccc}
\hline $\mathrm{I}(A)$ & $i_{1}$ & $i_{2}$ & $i_{3}$ & $i_{4}$ & $i_{5}$ & $i_{6}$ & $i_{7}$ & $i_{8}$ & $i_{9}$ & $i_{10}$ & $i_{11}$ \\
\hline 1 & 198.46 & -501.54 & -1.54 & 234.1 & -515.9 & 34.1 & 51.09 & -448.91 & -48.91 & -483.65 & 16.35 \\
2 & 173.87 & -526.74 & 73.62 & 133.27 & -366.86 & -16.74 & 302.54 & -597.46 & -197.46 & -609.39 & 140.61 \\
3 & -59.15 & -559.15 & 190.85 & 327.82 & --522.18 & -122.18 & 55.32 & -644.68 & 105.32 & -323.99 & -173.99 \\
4 & 193.82 & -706.55 & 193.74 & 4.82 & -395.31 & 104.89 & 334.47 & -615.53 & -315.53 & -533.17 & 17.04 \\
5 & 139.63 & -410.37 & -10.37 & 94.55 & -405.45 & 94.55 & 280.42 & -569.58 & -169.58 & -514.6 & 85.4 \\
6 & 208.81 & -592.20 & 8.60 & 250.68 & -600.22 & 50.49 & 177.00 & -623.00 & -123.00 & -636.07 & 63.93 \\
7 & 178.03 & -621.97 & 78.03 & 19.73 & -480.27 & 169.73 & 210.93 & -639.07 & -139.07 & -408.07 & -108.07 \\
8 & 205.03 & -544.97 & -44.97 & -27.94 & -527.94 & 272.06 & 210.29 & -689.71 & -89.71 & -387.37 & -137.37 \\
9 & 287.6 & -612.4 & -62.4 & 64.42 & -585.58 & 214.42 & 78.39 & -621.61 & -21.61 & -430.41 & -130.41 \\
10 & 201.35 & -448.65 & -23.65 & 195.86 & -404.14 & -29.14 & 38.85 & -461.15 & 38.85 & -436.06 & 13.94 \\
\hline
\end{tabular}

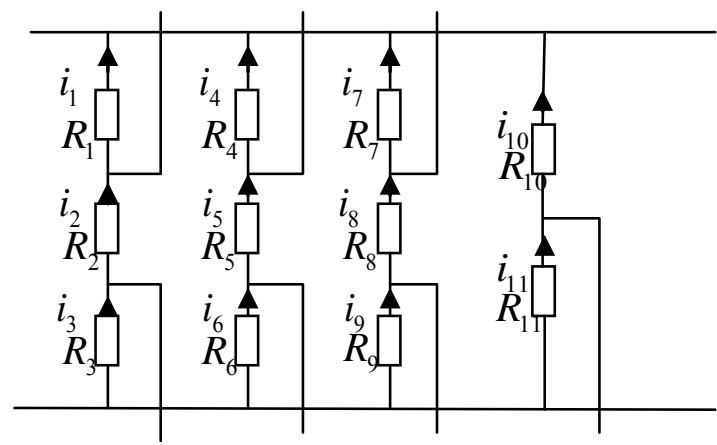

stablish equations by selecting condition 1 to condition 7. The comparisons of the results are shown in Table 2, in which the switch resistances have been converted into a ratio. As can be seen from the data in Table 2, this method can effectively calculate the relative value of switch resistance.

Figure 4. Circuit diagram of a $500 \mathrm{kV}$ switching station

TABLE II. COMPARISON OF THE SWITCH RESISTANCE BETWEEN THE STANDARD AND THE CALCULATED VALUES

\begin{tabular}{cccccccccccc}
\hline & $R_{1}$ & $R_{2}$ & $R_{3}$ & $R_{4}$ & $R_{5}$ & $R_{6}$ & $R_{7}$ & $R_{8}$ & $R_{9}$ & $R_{10}$ & $R_{11}$ \\
\hline$V_{\text {real }}$ & 1.00 & 1.50 & 1.30 & 1.80 & 2.00 & 1.60 & 1.50 & 1.30 & 1.00 & 1.20 & 1.50 \\
Vcal & 1.00 & 1.49 & 1.30 & 1.80 & 1.99 & 1.60 & 1.51 & 1.29 & 1.01 & 1.19 & 1.49 \\
$\operatorname{Err}(\%)$ & 0.00 & 0.67 & 0.00 & 0.00 & 0.50 & 0.00 & 0.67 & 0.77 & 1.00 & 0.83 & 0.67 \\
\hline
\end{tabular}

\section{CONCLUSIONS}

In this paper, evaluating the resistance value of the switching device is by measuring the real-time of the current flowing through the switching device. The equations were established by using the Kirchhoff'slaw and Ohm's law. Then select a reference switch to turn the homogeneous linear equations to nonhomogeneous ones. The enough number of equations is obtained by measuring the current conditions at different times. The simulation results show that the method can calculate the resistance of the switch effectively. So online assessment the status of the switch can achieve. The method is superior to the traditional off-line measuring method in whatever hardware costs or online assessment.

\section{REFERENCES}

[1] Li Kui, et al. Study on new measurement method of contact resistance[J]. Electric Switchgear, 1997,1(6):26-23(in Chinese).

[2] Aichi H, Tahara N. Analysis on the constriction resistance of the electric contact by the contact model using the electrolyte bath[C]. Proceedings of 20th International Conference on Electrical Contacts. 1994: 1-10.

[3] Niu Wenjing. Research of intelligent test of conductive loop resistance of circuitbreaker[D]. Dalian: Dalian University of Technology, 2004(in Chinese).

[4] Salamanca F, Borras F, Eggert H, et al. Preventive Diagnosis on High-Voltage Circuit Breakers[C]. CIGRE Symposium, 1993:120-02.

[5] Kumar Tyagi R, Singh Sodha N. Condition-based maintenance techniques for EHV-class circuit breakers[C]. Doble Client Conf, MA. 2001.

[6] Ohlen M, Dueck B. Wernli H. Dynamic Resistance Measurements-A Tool for Circuit Breaker Diagnostics[C].Stockholm Power Tech International Symposium on Electric Power Engineering. 1995, 6: 108-113.

[7] M.landry, A.Mercier, G.Ouellet, et al. A New Measurement Method of the Dynamic Contact Resistance of HV Circuit Breakers[J]. 
Transmission and Distribution Conference and Exhibition, 2006: 21-24

[8] Stanisic,Z, Megger Sweden AB, Stockholm, Sweden. Method for Static and Dynamic Resistance Measurements of HV Circuit Breaker[J].Innovative Smart Grid Technologies(ISGT Europe),2011: 1-5.

[9] Liu Guojin. Study on measurement of the contact resistance of electric apparatus $[\mathrm{J}]$. Electrical Measurement and Instrumentation, 2001, 38(422): 15-16(in Chinese).

[10] Xia Xiaofei. Research about the method that testing breaker loop resistance based on high impulse current. Electrical Technology[J],2013(10): 28-33(in Chinese).

[11] Niu Wenjing, Li Hongchun. Research about a new tester of the breaker contact resistance[J]. ElectroTechnical Journal, 2003: 4-6(in Chinese).

[12]Gao Fei, Li Hongchun, Zou Jiyan. Measurement of contact resistance of circuit breaker[J]. Electronic Instrumentation Customer, 2002, 9(5):36-38(in Chinese).
[13] Li Yanyan. On-line monitoring of HVCB[D]. Dalian: Dalian University of Technology, 2002:26-30(in Chinese).

[14] Jin Tao, Cai Chao, Wang Jun, Ruan Ling. Parameter calculation of high-voltage DC circuit breaker based on genetic algorithm[J]. Engineering Journal of Wuhan University, 2013, 46(2):223-227(in Chinese).

[15] Gong Wei. Research on on-line monitoring and state evaluating system for high voltage circuit breakers[D]. Liaoning: Northeastern University, 2008(in Chinese).

[16] Bian HaoWei. Study on on-line monitoring and fault diagnosis system for high voltage circuit breaker [D]. Yang Zhou: Yang Zhou University, 2012(in Chinese).

[17] Wang Junxia. The condition assessment method for HV circuit breakers based on FAHP[D]. Sichuan: Xihua University, 2013(in Chinese). 